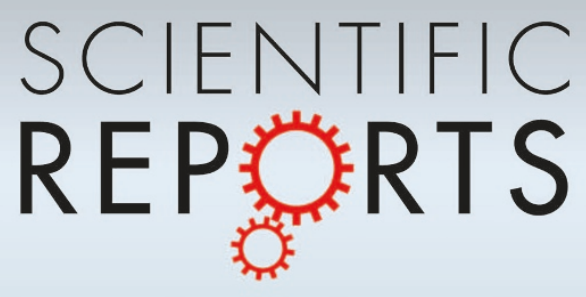

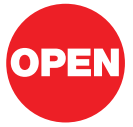

SUBJECT AREAS:

ELECTRICAL AND

ELECTRONIC

ENGINEERING

MECHANICAL ENGINEERING

SURFACE PATTERNING

APPLIED PHYSICS

Received

14 February 2013

Accepted

18 April 2013

Published

9 May 2013

Correspondence and requests for materials should be addressed to J.L. (jliu@mail.ipc.ac.

\section{Direct Desktop Printed-Circuits-on-Paper Flexible Electronics}

\author{
Yi Zheng ', Zhizhu He', Yunxia Gao' \& Jing Liu ${ }^{1,2}$ \\ ${ }^{1}$ Key Lab of Cryogenics and Beijing Key Lab of CryoBiomedical Engineering, Technical Institute of Physics and Chemistry, Chinese \\ Academy of Sciences, Beijing 100190, P. R. China, ${ }^{2}$ Department of Biomedical Engineering, School of Medicine, Tsinghua \\ University, Beijing 100084, P. R. China.
}

There currently lacks of a way to directly write out electronics, just like printing pictures on paper by an office printer. Here we show a desktop printing of flexible circuits on paper via developing liquid metal ink and related working mechanisms. Through modifying adhesion of the ink, overcoming its high surface tension by dispensing machine and designing a brush like porous pinhead for printing alloy and identifying matched substrate materials among different papers, the slightly oxidized alloy ink was demonstrated to be flexibly printed on coated paper, which could compose various functional electronics and the concept of Printed-Circuits-on-Paper was thus presented. Further, RTV silicone rubber was adopted as isolating inks and packaging material to guarantee the functional stability of the circuit, which suggests an approach for printing 3D hybrid electro-mechanical device. The present work paved the way for a low cost and easygoing method in directly printing paper electronics.

thas been widely known that the invention of printed circuits on boards (PCB) has brought about tremendous advances to modern electronics. Recently, with more concerns raised in the complicated and energyconsuming fabrication processes of conventional electronics, plentiful attentions have been made to find an additive way to make electronic components on any desired substrates, especially those flexible ones. Among the many efforts ever made, functional inks based on organic substances ${ }^{1,2}$, polymers ${ }^{3,4}$, nanoparticles ${ }^{5-7}$, thin-film semiconductors ${ }^{8,9}$ etc. have been developed for manufacturing flexible electronics, which significantly contributed to the development of antennas ${ }^{10,11}$, transistors ${ }^{12,13}$, solar cells ${ }^{14,15}$, radio frequency identification (RFID) $)^{16}$, flexible displays ${ }^{17-19}$, electronic clothes ${ }^{20}$, sensors ${ }^{21,22}$ and so forth. For developing various promising flexible substrate materials, paper is specially being paid with considerable attentions ${ }^{23-26}$ in printed electronics owing to its excellent properties such as easy accessibility, low-cost, foldability, disposability, retrievability and nonpollution. Meanwhile, enormous efforts have also been made to find highly conductive inks ${ }^{27-32}$ so as to perform direct writing of flexible circuit. So far, many currently available electrical inks are still not conductive enough. To further improve their electrical conductivity, loading nanoparticle to the base material is an important approach. However, some basic issues still remain. For instance, such composite inks' conductivity is heavily dependent on the particles' loading ratio and bonding mechanism after solidification. However, a difficulty lies in is that the loading ratio of particles in solution is generally low. In addition, preparation of such conductive inks is still somewhat complicated. For some ink materials, it has to adopt relatively high post-processing temperature (for example $400^{\circ} \mathrm{C}$ ) to improve their conductivity, while most plastic and paper substrates require processing temperature below $150^{\circ} \mathrm{C}$. So far, there still lacks of a reliable way which is capable of directly writing out electronic circuits, just like printing a picture on paper via a desktop printer in office.

Recently, the room temperature liquid metal or its alloys was found to own very interesting properties as printing inks especially in flexible electronics. Along this way, a direct approach to immediately write out electronics through such inks is incubating ${ }^{33,34}$. This affordable processing appears rather succinct and efficient. However, there is currently lacking of an automatic printing method for the increasing use of such promising technology which is a key towards pervasive consumer electronics. Clearly, the manual writing approach as demonstrated before would restrict the practical value of the liquid metal printed electronics after all. Therefore, an automatic and direct printing of electronics by developing a desktop printer with intelligent-controlling ability and high-precision is urgently needed to fulfill such requirement.

Here we show an entire solution for automatic and direct printing of liquid metal ink on paper, through resolving a series of important issues, such as modifying the adhesion of the alloy inks, overcoming the high surface tension of the ink via the dispensing machine (Fig. 1) and designing a brush like porous pinhead for printing liquid alloy inks and identifying the matched substrate materials among different types of papers. The 


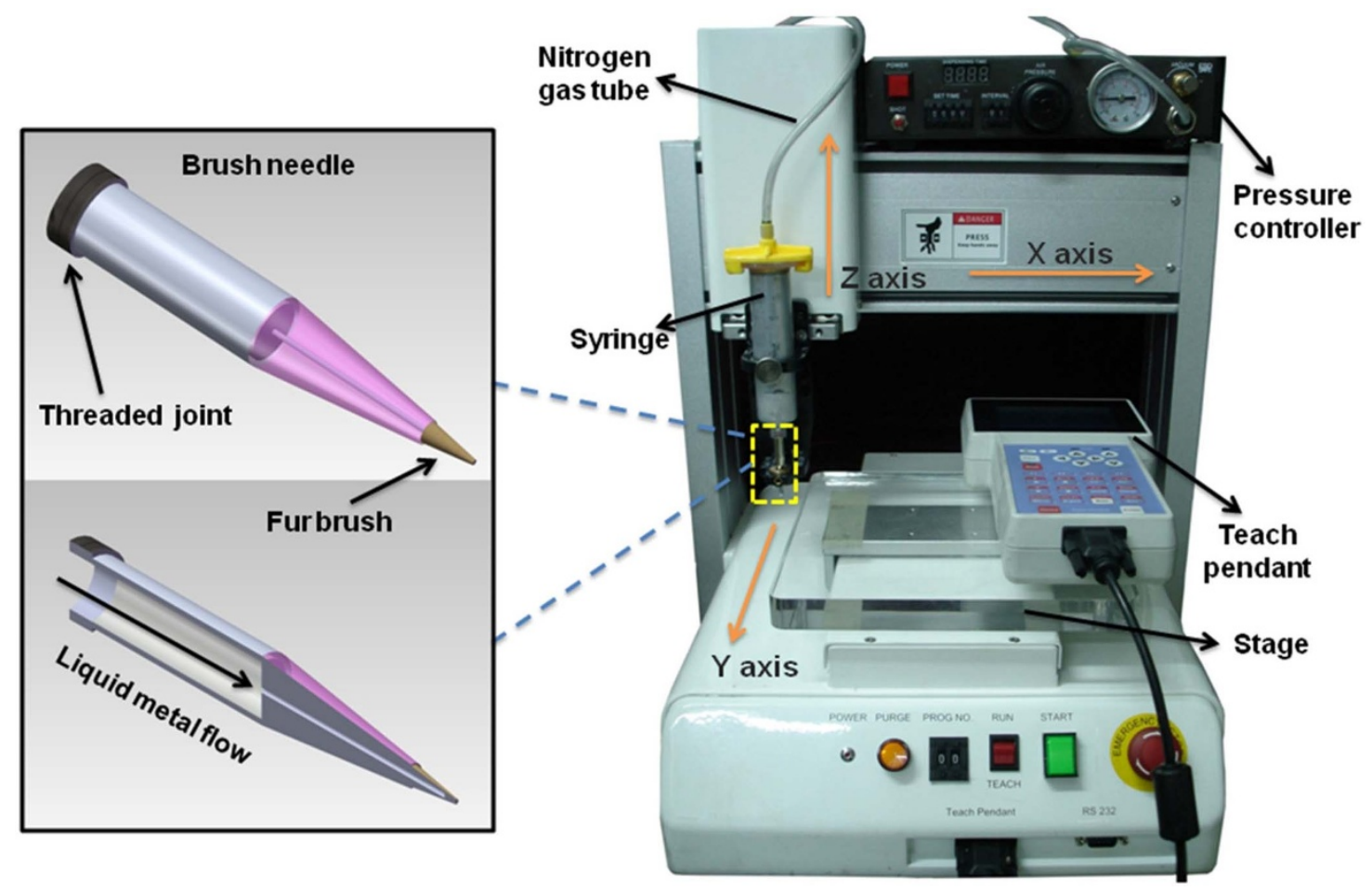

Figure $1 \mid$ Schematics for experimental printing pinhead and desktop printer setup.

prototyping desktop printer can be manipulated with ease to print out various patterns on the selected papers as desired under control of the computer software. Particularly, paper with advantages of flexibility, low-cost and recyclability was investigated as a highly suitable substrate material for printing electronics. This may lead to the concept of Printed-Circuits-on-Paper (PCP). Further, the room temperature vulcanized silicone rubber which has been widely utilized in electronics packaging was adopted as isolating inks so as to print them out as encapsulated structure for packaging the liquid metal circuits and making a multistory hybrid electro-mechanical structure. Through these efforts, various typical circuits and components have been successfully printed on coated paper, including conductive wires, 3D conductor structure, inductance coil and flexible antenna etc.

\section{Results}

Printing papers. To evaluate the compatibility between the liquid metal ink and the paper substrate, a high-speed camera was utilized to record the gliding process of the liquid alloy droplet on various paper substrates with a uniform slope angle of $30^{\circ}$. To ensure the fluid feature, high-purity liquid alloy $\mathrm{GaIn}_{24.5}$ which was not deliberately oxidized was used. For comparison purpose, droplets with same volume should pass through the same distance starting from a standstill state. Therefore, an injector pump with a speed of $10 \mathrm{ml} / \mathrm{h}$ was used to generate droplets so as to ensure same droplet volume and timing starting from the moment of gliding. Figure 2 shows the detailed gliding process of liquid alloy ink droplet on coated paper. Here, droplets on all papers have run the same distance as $60 \mathrm{~mm}$. Figure 3 presents the distance (s) as a function of detection time and the internal schematic diagram depicts the force analysis of droplet on paper over the process. It is known that there are mainly three constant forces acting on the droplet, namely gravity $(\mathrm{G})$, supporting force $(\mathrm{N})$ and resistance (f). Droplets are considered to run uniformly accelerated linear motion along papers under the effects of forces. The classical
Newton second law and uniformly accelerated linear motion law are suitable to describe such motion which are presented as follows:

$$
\begin{gathered}
s=\frac{1}{2} a t^{2} \\
G_{1}-f=m a
\end{gathered}
$$

where, $s$ is the distance along paper, $t$ is the detection time of gliding, $G_{1}$ is the gravity component which is parallel to the inclined paper substrate; $\boldsymbol{f}$ is the resistance force containing the friction and adhesion force; $\boldsymbol{m}$ and $\boldsymbol{a}$ are the quality and acceleration of droplet, respectively. It can be seen from the curves that the relationship of $s$ and $\boldsymbol{t}$ fits well with the equation (1). And according to equation (2), the acceleration can be described as:

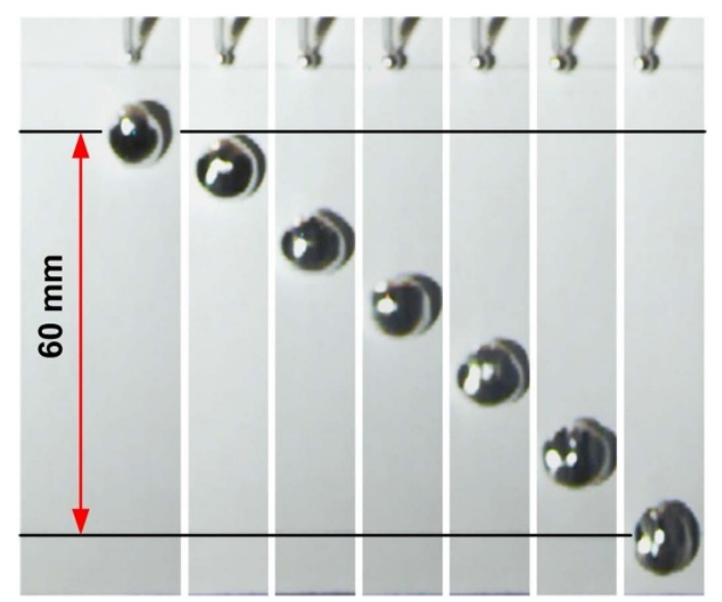

Figure $2 \mid$ Sequential optical images of liquid metal droplet gliding along the coated paper with a slope angle of $30^{\circ}$ which were recorded by highspeed camera. 


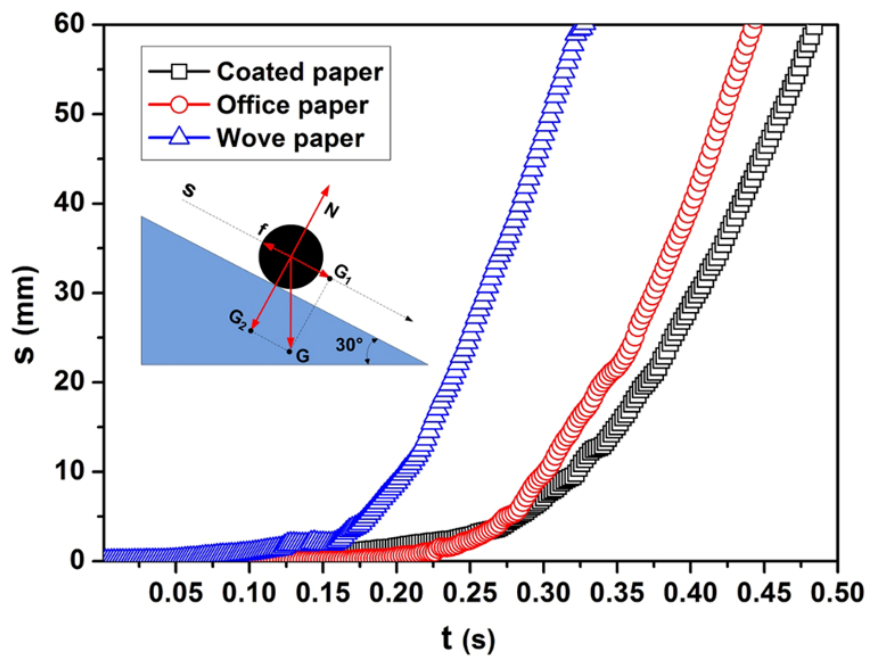

Figure $3 \mid$ Distance (s) of droplets as a function of measured time (t) on coated paper, office paper, wove paper, respectively. The inset shows the sketch map of force analysis of the droplet in the gliding process.

$$
a=\frac{G_{1}-f}{m}=\frac{m g \sin \theta-f}{m}=g \sin \theta-\frac{f}{m}
$$

where, $\theta$ is the slope angle between paper substrates and horizontal line. Additionally, for all paper types, parameter $\theta, g$ and $m$ are all constant values. Therefore, there are only two variables in the equation (3), $a$ and $f$. From Fig. 3 , it can be found that acceleration on coated paper is the minimum of all, which indicates that it has maximum resistance force on the surface of coated paper. In order to clarify the surface roughness of three papers, we have performed an atomic force microscope (AFM) analysis. Figure 4A, B and C present the AFM images of surface morphology of wove paper, office paper and coated paper, respectively. The measured average $\mathrm{Rq}$ of wove paper, office paper and coated paper is $120.8 \mathrm{~nm}, 70.7 \mathrm{~nm}$ and $12.9 \mathrm{~nm}$, respectively. It has been widely accepted that the rougher the surface, the larger the friction, which means coated paper has the minimal surface friction. Conclusions regarding the numerical relations can be obtained as follows:

$$
\begin{array}{ll}
\text { Total resistance : } & f_{\text {coated }}>f_{\text {office }}>f_{\text {wove }} ; \\
\text { Surface friction : } & f_{\text {wove }}>f_{\text {office }}>f_{\text {coated }} ; \\
\text { Adhesive force : } & f_{\text {coated }}>f_{\text {office }}>f_{\text {wove }} .
\end{array}
$$

Therefore, coated paper possesses the maximal adhesive force, indicating that coated paper has the best compatibility with liquid $\mathrm{GaIn}_{24.5}$ based alloy ink among all applied papers.

Coated paper with high smoothness and brightness is an advanced paper substrate which has been commonly used in printing book cover, elegant advertisements, commodity packaging, and labels, etc. It is mainly manufactured by coating a pigment compounds layer on base-paper. Conventional filler pigments mainly contain ground calcium carbonate (GCC), precipitated calcium carbonate aragonite (PCC) and Kaolin (mainly $\mathrm{Al}_{2} \mathrm{O}_{3} \cdot 2 \mathrm{SiO}_{2} \cdot \mathrm{H}_{2} \mathrm{O}$ ), as well as chemical additives such as dispersants, resins, sodium polyacrylate (NaPA), and poly (styrenebutadiene) (SB) latex binder etc. Investigations have been conducted to confirm that addition of the the above-mentioned fillers has a positive effect on the adhesion of paper $^{35-37}$. In order to further disclose the mechanism for the surface adhesive, an energy dispersive spectroscopy (EDS) analysis was also conducted to survey the surface elements of the tested coated paper. Figure 5 exhibits the weight ratio of various elements on the surface of the coated paper. It can be seen that oxygen and silicon elements occupy a large proportion among all elements. As a result, combined with the former efforts, a better compatibility between paper and ink can be obtained through properly oxidizing $\mathrm{GaIn}_{24.5}$ alloy ink.

Automatic printing of circuits on coated paper. Automatic printing of $\mathrm{GaIn}_{24.5}$ based inks on coated paper was implemented utilizing the developed system, and the direct printing process was illustrated in Fig. 6. Through the evaluation as performed above, here the commercially available coated paper (with a weight specification of $200 \mathrm{~g} / \mathrm{m}^{2}$ ) was adopted as the printing substrate. Apparatus equipped with single printing syringe $A$ or $B$ were utilized and the structure materials of matched printing needle were porous brush and orifice metal, respectively. In addition, syringe A and B were respectively loaded with $\mathrm{GaIn}_{24.5}$ ink and RTV silicone rubber. During the printing process, syringe A was firstly installed to print required patterns on coated paper according to the digitalized program, and then overprinted by RTV silicone rubber from syringe $\mathrm{B}$. When necessary, multilayer circuit structures can be fabricated by sequentially printing liquid metal circuitries bypassing the upper surface of the cured silicone rubber. In this way, a three dimensional (3D) hybrid electro-mechanical device on paper can be quickly printed out.

Several typical directly printed circuits containing bending circuit with regularity (1), packaged straight wires (2), circuit nodes (3), stereoscopic flexible circuits (4) and annulus wire with LED (5) on coated paper are presented in Fig. 7(A), which illustrate the excellent adhesion between $\mathrm{GaIn}_{24.5}$ based ink and coated paper, as well as outstanding elastic characteristics of this kind of RTV silicone

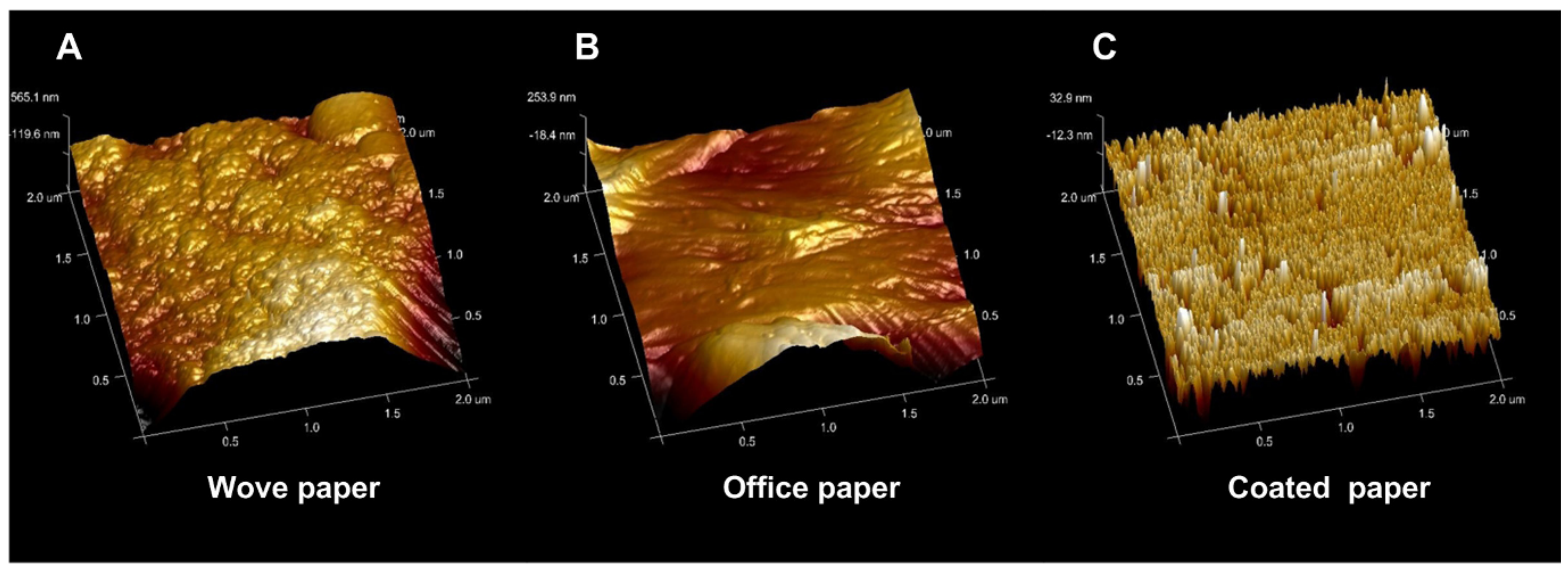

Figure $4 \mid$ Surface morphologies of three typical papers. AFM images $\left(2 \times 2 \mu \mathrm{m}^{2}\right)$ of surfaces of Wove paper (A), Office paper (B) and Coated paper (C), respectively. 


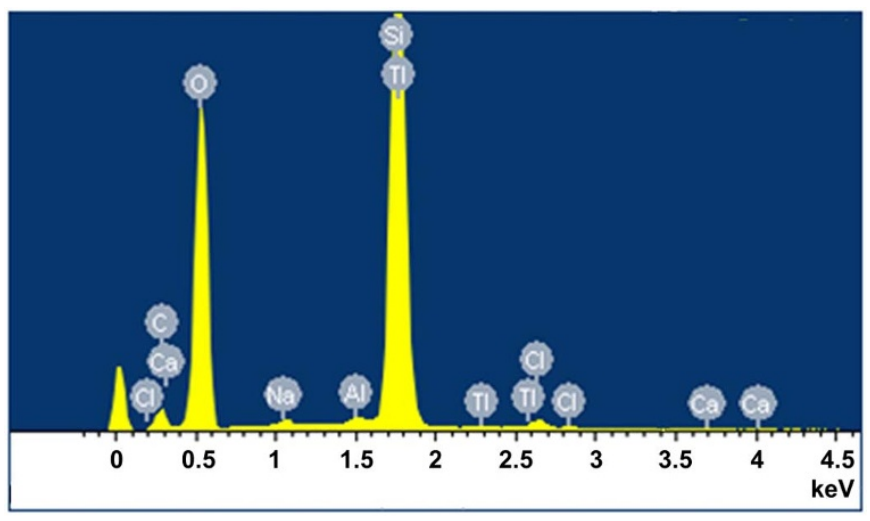

Figure 5 | Energy dispersive spectroscopy (EDS) picture of elements on coated paper surface.

rubber. More printed functional electronics including inductance coil (1) and RFID (2) antenna are given in Fig. 7(B), and picture 3 reveals the excellent flexibility of the fabricated antenna after packaging. Experimental results confirm that the cured silicone rubber can commendably prevent the liquid ink from flowing along the wires, which to a large extent will contribute to the functional stability of the printed circuits or electrical components.

It is well known that conductive inks of conventional printed electronics usually function after solidifying. This indicates that the fabricated electronics have mechanically flexible restriction. However, the present ink remains liquid under functional conditions after being encapsulated by RTV silicone rubber, while the substrate and the elastic rubber form a channel to maintain the electrical structure. Therefore, the fabricated circuits cannot be broken off easily even under frequent bending, showing an attractive and distinguished mechanical flexibility which is a critical advantage in fabricating flexible electronics. Moreover, the well-encapsulated liquid alloy ink on paper can be conveniently and responsibly stored and carried. Overall, the present approach constitutes a vital step towards truly directly printed electronics.

Figure 8A presented the physical images of the printed circuit with a width of $1.5 \mathrm{~mm}$ on coated paper, and Fig. $8 \mathrm{~B}$ and $\mathrm{C}$ gave the SEM graphs of surface and cross-sectional topography of the printed liquid wires respectively. It can be discovered from the images that the liquid metal wire is adhered closely to the surface of the paper which has a multi-groove structure. The directly printed liquid circuit on coated paper is relatively uniform and the thickness is about $120 \mu \mathrm{m}$. Simultaneously, the SEM graphs prove that $\mathrm{GaIn}_{24.5}$ based ink possesses wonderful adhesion with coated paper substrate once again, indicating that the compatibility between the ink and coated paper is functionally favorable. Apparently, processing should be further advanced to fabricate thinner even nanoscale circuit to apply in highly required integrated electronics. This needs new efforts in the near future.

Electrical reliability of flexible circuit as a function of bending angles. As an innovative ink with tremendous potentials to be further applied in future flexible electronics manufacturing, the resistance stability of the liquid metal before and after bending is vital. Here, a digital bridge with an equipment deviation of $0.003 \Omega$ was employed to measure the resistance variation of liquid metal circuit when folding to five angles. Liquid metal circuit whose length and width is about $18 \mathrm{~cm}$ and $1 \mathrm{~mm}$ respectively was printed. Two copper wires with the same resistance of $0.0275 \Omega$ were applied to serve as measuring electrodes. The printed circuits were well packaged by RTV silicone rubber and the operating frequency and voltage of digital bridge was set to $1.0 \mathrm{kHz}$ and $1 \mathrm{~V}$, respectively. Figure 9 shows the resistance results according to the measurements. It is necessary to point out that all the measured resistances are the total values that contain the resistances of both circuit and copper electrodes, and in order to reduce the deviation, all resulting values in picture are an average of 3 measured values. It can be evidently observed that resistances vary just in a limited range, which indicates that the well-encapsulated printed liquid metal wire on paper could meet well the requirement of the flexible circuit. Meanwhile, the liquid silicone rubber scarcely affects the electrical performance of the printed liquid circuit. This is rather beneficial for the wide adaptability of PCP.

\section{Discussion}

In this article, an innovative approach to automatically fabricate electronics by directly printing liquid alloy based inks on coated paper is described. Compared with the conventional ways of making flexible electronics using existing conductive inks, the present highefficiency direct printing process has relatively lower-cost, simpler process, well acceptable conductivity and is more environmentally friendly which guarantees the really "green" fabrication of electronics. Moreover, the whole printing process is completed under room temperature, which hardly brings about detrimental influence to the paper substrate and is capable of ensuring the printed circuits' own excellent electrical performance and mechanical flexibility. Further, the recycling of metal inks is easier to be completed just by properly destroying the external elastic silicone rubber and then efficiently reclaiming the noble material by $30 \% \mathrm{NaOH}$ solution. Finally, liquid metal ink runs in liquid state which indicates that liquid metal circuits printed on flexible substrates will not be easily fractured owing to its unique liquidus property.

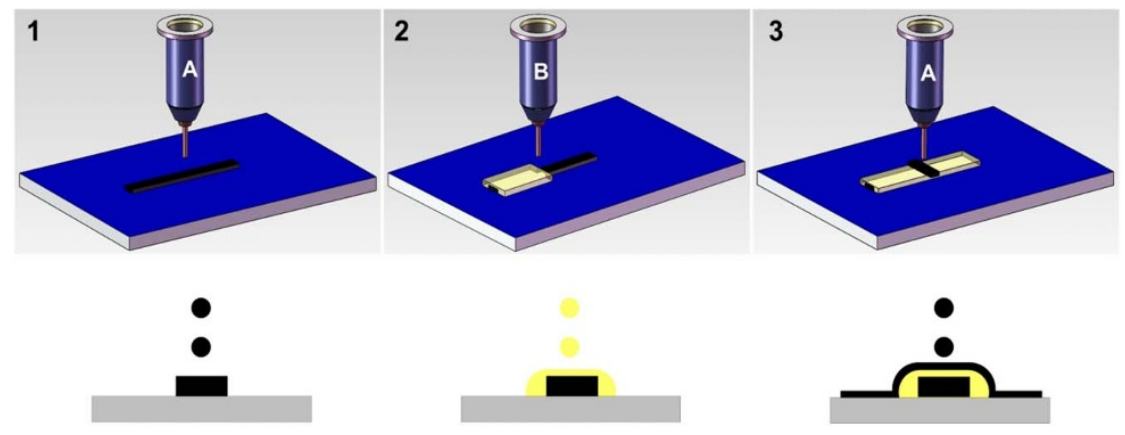

Figure 6 Schematic of liquid alloy printing of electrical circuitry on coated paper. Liquid metal (A) is firstly printed on paper (Step 1); Then RTV silicone rubber $(B)$ is overprinted sequentially as encapsulated material (Step 2); When necessary, a multilayer circuit structure is fabricated by printing liquid metal (A) circuitry bypassing the upper surface of cured silicone rubber (Step 3). 

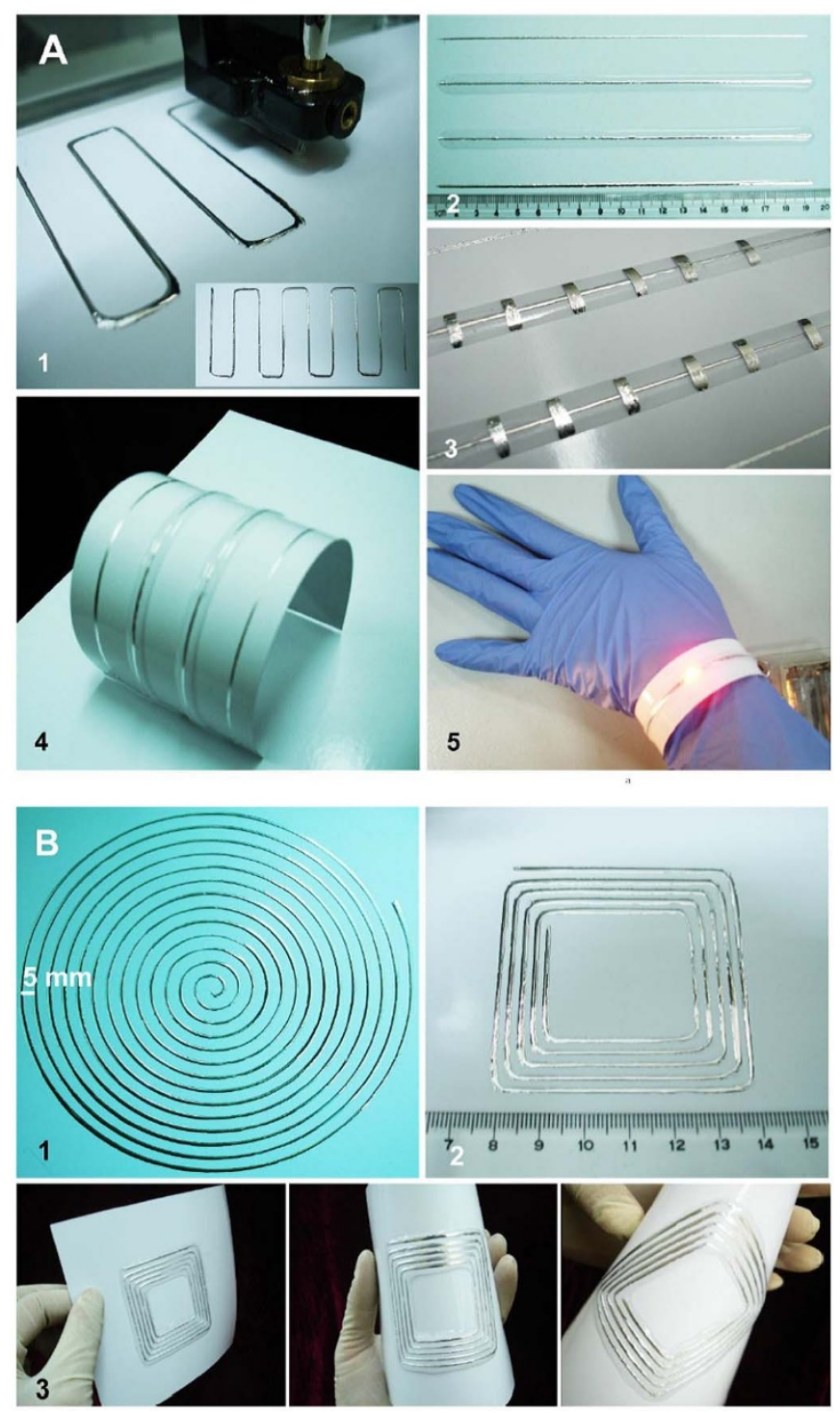

Figure $7 \mid$ Desktop printed electronic components. (A) Physical diagram of $\mathrm{GaIn}_{24.5}$ based liquid alloy directly printed on coated paper by a dispenser printer and optical images for various conductive wires on coated paper: (1) Manufacturing process of printed electronics. The inset shows the regular bending circuit; (2) Metal conductor lines covered with silicone rubber; (3) Multi-layer structure or electrical nodes; (4) Threedimensional structure of printed conductor on paper; (5) Galvanical annulus wire attached with LED. (B) Optical images for printed functional components on coated paper: (1) Inductance coil; (2) RFID antenna;

(3) Presentation of prominent flexibility of printed electronics.

As disclosed by former research ${ }^{33}$, the content of the oxides in liquid alloy can efficiently alter the viscosity of the ink which greatly affects the final performance of the circuits and components printed directly on papers. The adhesion of alloy ink can be upgraded through the process of oxidizing, as well as altering the weight ratio of the chemical composition. Also, the surface adhesion of the coated paper materials can be modified by ultraviolet-C ray radiation (UVC Irradiation $)^{35}$. Furthermore, the coated paper substrate can also be simply manufactured on one's own as needed ${ }^{36,37}$. And reinforced RTV silicone rubbers or other alternative adhesives can be applied to substitute this basic encapsulated material for extreme working condition. In the near future, more liquid metals or alloys can be developed as suitable inks to be directly printed on various compatible papers. Clearly, combined with more functional materials such

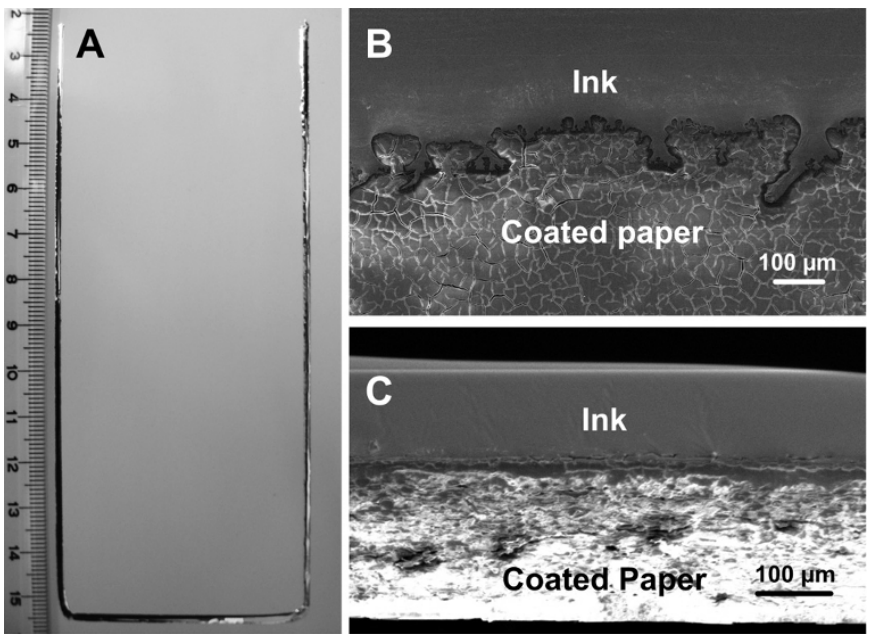

Figure $8 \mid$ Characterization of the printed conductive objects. (A) Optical image of printed circuit on coated paper with a width of $1.5 \mathrm{~mm}$. (B) and (C) are SEM images of surface and cross-section of printed liquid metal ink on coated paper.

as conductive polymers, metal nanoparticles or semiconductors, further performance modification of such inks is likely to be realized to meet future high profile needs in a wide variety of flexible electronics. It is expected that such unique approach will be applied in a sufficient number of electrical areas including rapid-prototyping circuits (e.g., E-Card), integrated circuit on paper, sensors, 3D mechanical switches and antennas, electronic solar cell arrays, functional RFID tags on books, even daily life personal electronics and educational training.

However, as a newly emerging technology, the present method also raised a series of practical issues waiting to be further better solved such as guaranteeing an ever higher resolution circuital uniformity as well as more rapid printing speed of the process. In this side, considerable researches as implemented in our work disclosed that, through resolving the printing challenge caused by the large surface tension of GaIn24.5 ink (via oxidation and searching compatible paper substrate), the commonly encountered troubles such as frequent occurrence of the intermittent structure and droplet formation of conductive line were successfully overcome. In addition,

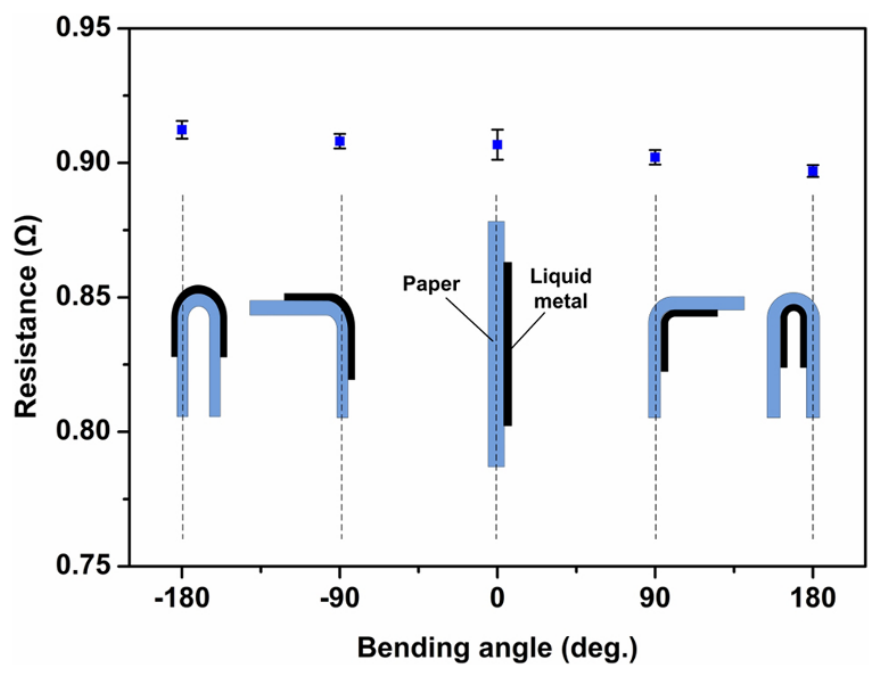

Figure 9 | Resistance values of liquid circuit at five different bending angles. 
better circuital uniformity and resolution can still be possible in the coming time subject to further endeavor. Along this direction, several potential strategies such as improving hardness of the fur brush, altering the length of fur, identifying ideal distance between pinhead and paper substrate, adjusting the pressure of driving gas via the controller or developing more advanced pinhead can be applied to fabricate smoother and narrower circuits. In addition, more paper materials should also be tried as substrate materials so as to vigorously advance the PCP technology.

In summary, the present method offers a vital opportunity to realize rapid fabrication of inexpensive, disposable, conveniently portable circuits and components, which paved a way towards desktop direct printed electronics. With designed software, required functional electronic circuits can be automatically printed on paper just by clicking the corresponding specific code or pictures as people usually do in office. This will extensively stimulate tremendous efforts in either fundamental research or practical applications of $\mathrm{PCP}$ in the coming time.

\section{Methods}

Preparation of ink materials. The flowable and highly conductive room temperature liquid metals or alloys at normal pressure and temperature are a kind of newly emerging functional materials which were significantly neglected before. Several easily available metals or alloys such as gallium, indium, GaIn ${ }_{10}$, and $\mathrm{GaIn}_{24.5}$ etc. all show very promising features in flexible electronics. However, a critical issue to impede their extensive applications in printed electronics is the large surface tension of the liquid metal ink and the poor adhesion thus caused, as well as lacking of an appropriate desktop printing machine. In our former efforts, oxidation on the metal ink was identified as an efficient approach to significantly modify the adhesion of liquid metals on various printing substrates. Therefore, $\mathrm{GaIn}_{24.5}$ alloy with a melting point of $16^{\circ} \mathrm{C}$ was selected here as the testing ink. For this purpose, high-purity gallium and indium (with purity of 99.99 percent) metals as source materials were weighted with a ratio of $75.5: 24.5$ in line with the chemical compositions of $\mathrm{GaIn}_{24.5}$ alloy. The total processes were generalized as follows: Firstly, configuring the eutectic $\mathrm{GaIn}_{24.5}$ alloy. The weighted gallium and indium metals according to the required percentage are mixed in the beaker which was syringed by deionized water in advance and heated to about $50^{\circ} \mathrm{C}$ until metals were fused completely, then stirred slightly. Secondly, oxidizing the liquid metal, the well-configured alloy was stirred for a required period using magnetic stirrer at a moderate rotational speed at room temperature so as to obtain $\mathrm{GaIn}_{24.5}$ based alloys with proper oxygen content.

According to our previous experiments ${ }^{33}$, it is rather worthwhile to note that the adhesive ability and electrical resistance of liquid alloy are related with oxygen content. Adhesive ability improves with the increase of the oxygen content, but electrical resistance only increases slightly. This indicates that such stirring method could hardly affect the electrical performance of the metal ink which is a beneficial merit for its practical use. The stirring time and rotational speed can be controlled to alter the oxygen ratio of the alloys as needed. In this work, the liquid metal ink is required to possess excellent adhesive ability to be easily printed on paper. However, for liquid metal, a larger wettability or viscosity always means poorer flowability which is detrimental for the ink to flow through the porous structured printing pinhead. Therefore, oxygen content with suitable ratio by weight is rather crucial to make alloy ink. Through a series of testing, $\mathrm{GaIn}_{24.5}$ based alloys with oxygen content of about 0.1 wt\% which appears partial sticky was found to be a kind of suitable printed ink.

Identification of matched papers for printing liquid metal ink. Paper substrates, as promising materials for fabricating printed electronics, offer plentiful particularities that are completely different from the properties of polydimethylsiloxane (PDMS) used in conventional flexible PCB field. So far, paper has been regarded as one of the cheapest and most extensively used flexible substrates in everyday life owing to its low-cost and favorable portability. In addition, paper materials with excellent flexibility can be easily folded, twisted and even bended for storage in limited space or to conveniently and speedily form spatial self-standing structures. Moreover, paper generally owns considerably thinner size and lighter weight than conventional circuit platforms ${ }^{24}$. Last but not the least, paper is definitely a kind of "green" material that is recyclable and made of renewable raw materials.

Our experiments found that the compatibility between liquid metal ink and paper substrates is a critical issue significantly affecting the printing quality and the functional PCP thus made. In addition to the modification of ink, the adhesion properties of various paper substrates should also be paid with enough attentions. For the PCP, various commercially available paper materials including office paper, wove paper and coated paper (with a weight specification of $200 \mathrm{~g} / \mathrm{m}^{2}$ ) have been experimentally evaluated as printing mediums. Through a group of comparative tests, we found that only the coated paper can embody an excellent compatibility with the well-prepared alloy ink. Therefore, focus has been paid to confirm and find out the intrinsic cause of this discovery in the present paper.
Printable isolation ink for packaging material. Considering the liquidus properties of the current metal ink, when exposed in air, the fabricated patterns are apt to be changed. For the purpose of simplifying, protecting, insulating and stabilizing the printed liquid circuits, appropriate packaging on such PCP is very necessary. To date, liquid silicone rubber (LSR) is increasingly utilized in electronics packaging because of its excellent weatherability and thermal stability, oxidation resistance, lowtemperature flexibility, and good dielectric properties. Along this way, a kind of commercially available and low-cost single component room temperature vulcanizing (RTV) silicone rubber (Type 705, NAN DA, Liyang Kangda Chemical Co. Ltd, Jiangsu Provence, China) was introduced as printable packaging ink and demonstrated as an excellent basic packaging material herein. Such kind of rubbers can be applied (liquid state) and cured (elastic state) at room temperature through automatically absorbing moisture from the air without any heat treatment, and no environmentally harmful gas is discharged. Clearly, through combination of the flowable electrical and insulating inks, even a three-dimensional (3D) electronics device can be printed out using the present method, such that by printing inks on the surface of the cured elastomer under control of digitized program.

Prototyping desktop printer and printing pen. A commercially available rubber dispenser (TYSR-200D, Tinyo Electronics Corporation, Beijing, China) was modified to realize the present automatic printing. For the printing pinhead, a brush like porous needle pen was developed to contain liquid metal ink inside and then print it on the paper. Both structures of the desktop printing apparatus and cross-section of the pinhead (inner diameter $0.51 \mathrm{~mm}$ ) whose cusp is fur material are shown in Fig. 1. It is worth noting herein that according to various application requirements, the printing speed is adjustable by inputting speed setting program via the teach pendant which can be set in a range of $0.1 \sim 400 \mathrm{~mm} / \mathrm{s}^{26}$. In view of facilitating the printing quality, the printing speed and pressure herein were set at $10 \mathrm{~mm} / \mathrm{s}$ and $10-30$ psi, respectively. Such performance could serve well the need of PCP technology. Further improvement of the printing speed can be made in the near future. In this research, the wellprepared $\mathrm{GaIn}_{24.5}$ ink in syringe was pushed into the porous brush needle through nitrogen gas with prescribed pressure which can be regulated via the controller. Then requisite liquid metal patterns were printed automatically on paper via the fur brush under control of corresponding digitized program embedded in the teach pendant. Afterwards, RTV silicone rubber loaded in another printing syringe is controlled to overprint the liquid metal wires for the purpose of insulation and encapsulation.

1. Forrest, S. R. The path to ubiquitous and low-cost organic electronic appliances on plastic. Nature 428, 911-918 (2004).

2. Parashkov, R., Becker, E., Riedl, T., Johannes, H. H. \& Kowalsky, W. Large area electronics using printing methods. Proc. IEEE 93, 1321-1329 (2005).

3. Yoshioka, Y. \& Jabbour, G. E. Desktop inkjet printer as a tool to print conducting polymers. Synth. Met. 156, 779-783-213 (2006).

4. de Gans, B.-J., Duineveld, P. C. \& Schubert, U. S. Inkjet printing of polymers: State of the art and future developments. Adv. Mater. 16, 203-213 (2004).

5. Fuller, S. B., Wilhelm, E. J. \& Jacobson, J. M. Ink-jet printed nanoparticle microelectromechanical systems. J. Microelectromech. S. 11, 54-60 (2002).

6. Hwang, J. K. et al. Direct nanoprinting by liquid-bridge-mediated nanotransfer moulding. Nat. Nanotechnol. 5, 742-748 (2010).

7. Yu, R. et al. Piezotronic effect on the transport properties of $\mathrm{GaN}$ nanobelts for active flexible electronics. Adv. Mater. 24, 3532-3537 (2012).

8. Sun, Y. \& Rogers, J. A. Inorganic semiconductors for flexible electronics. $A d v$. Mater. 19, 1897-1916 (2007).

9. Baca, A. J. et al. Semiconductor wires and ribbons for high-performance flexible electronics. Angew. Chem. Int. Ed. 47, 5524-5542 (2008).

10. Adams, J. J. et al. Conformal printing of electrically small antennas on threedimensional surfaces. Adv. Mater. 23, 1335-1340 (2011).

11. Perelaer, J., de Gans, B.-J. \& Schubert, U. S. Ink-jet printing and microwave sintering of conductive silver tracks. Adv. Mater. 18, 2101-2104 (2006).

12. Jurchescu, O. D. et al. Correlation between microstructure, electronic properties and flicker noise in organic thin film transistors. App. Phys. Lett. 92, 132103-1-3 (2008).

13. Jurchescu, O. D., Popinciuc, M., van Wees, B. J. \& Palstra, T. T. M. Interfacecontrolled, high-mobility organic transistors. Adv. Mater. 19, 688-692 (2007).

14. Rowell, M. W. et al. Organic solar cells with carbon nanotube network electrodes. App. Phys. Lett. 88, 233506-1-3 (2006).

15. Hoth, C. N., Choulis, S. A., Schilinsky, P. \& Brabec, C. J. High photovoltaic performance of inkjet printed polymer: Fullerene blends. Adv. Mater. 19, 39733978 (2007).

16. Hornyak, T. RFID powder. Sci. Am. 298, 68-71 (2008).

17. Zhou, L. et al. All-organic active matrix flexible display. App. Phys. Lett. 88 , 083502-1-3 (2006)

18. Wakehama, S. J. et al. Low temperature remote plasma sputtering of indium tin oxide for flexible display applications. Thin Solid Films 518, 1355-1358 (2009).

19. Yoon, B. et al. Inkjet printing of conjugated polymer precursors on paper substrates for colorimetric sensing and flexible electrothermochromic display. Adv. Mater. 23, 5492-5497 (2011).

20. Yamada, T. et al. A stretchable carbon nanotube strain sensor for human-motion detection. Nat. Nanotech. 6, 296-301 (2011).

21. Sun, Y. \& Wang, H. H. High-performance, flexible hydrogen sensors that use carbon nanotubes decorated with palladium nanoparticles. Adv. Mater. 19, 28182823 (2007). 
22. Zirkl, M. et al. An all-printed ferroelectric active matrix sensor network based on only five functional materials forming a touchless control interface. Adv. Mater. 23, 2069-2074 (2011).

23. Tao, H. et al. Metamaterials on paper as a sensing platform. Adv. Mater. 23, 31973201 (2011).

24. Siegel, A. C. et al. Foldable printed circuit boards on paper substrates. Adv Funct. Mater. 20, 28-35 (2010).

25. Rogers, J. A. Electronics: A diverse printed future. Nature 468, 177-178 (2010).

26. Tobjörk, D. \& Österbacka, R. Paper electronics. Adv. Mater. 23, 1935-1961 (2011).

27. Sun, Y. \& Xia, Y. Shape-controlled synthesis of gold and silver nanoparticles. Science 298, 2176-2179 (2002).

28. Pyatenko, A., Yamaguchi, M. \& Suzuki, M. Synthesis of spherical silver nanoparticles with controllable sizes in aqueous solutions. J. Phys. Chem. C. 111, 7910-7917 (2007).

29. Russo, A. et al. Pen-on-paper flexible electronics. Adv. Mater. 23, 3426-3430 (2011).

30. Aernouts, T. et al. Printable anodes for flexible organic solar cell modules. Thin Solid Films 451-452, 22-25 (2004).

31. Eom, S. H. et al. Polymer solar cells based on inkjet-printed PEDOT: PSS layer. Org. Electron. 10, 536-543 (2009).

32. Kim, N. S. \& Han, K. N. Future direction of direct writing. J. Appl. Phys. 108, 102801-1-6 (2010).

33. Gao, Y., Li, H. \& Liu, J. Direct writing of flexible electronics through room temperature liquid metal ink. PLoS ONE 7, e45485 (2012).

34. Li, H., Yang, Y. \& Liu, J. Printable tiny thermocouple by liquid metal gallium and its matching metal. App. Phys. Lett. 101, 073511-1-3 (2012).

35. Määttänen, A. et al. Enhanced surface wetting of pigment coated paper by UVC irradiation. Ind. Eng. Chem. Res. 49, 11351-11356 (2010).
36. Bollström, R. et al. A multilayer coated fiber-based substrate suitable for printed functionality. Org. Electron. 10, 1020-1023 (2009).

37. Chen, W., Tang, X., Considine, J. \& Turner, K. T. Effect of inorganic fillers in paper on the adhesion of pressure-sensitive adhesives. J. Adhes. Sci. Technol. 25, 581-596 (2011).

\section{Acknowledgments}

The authors acknowledge constructive suggestions from Prof. Yixin Zhou of Technical Institute of Physics and Chemistry (TIPC), Chinese Academy of Sciences (CAS). This work is partially supported by the TIPC of CAS.

\section{Author contributions}

Y.Z. designed and performed the experiments, and wrote the manuscript.Z.Z.H. and Y.X.G discussed part of the results. J.L. conceived the present work, designed the experiments and wrote the manuscript.

\section{Additional information}

Competing financial interests: The authors declare no competing financial interests.

License: This work is licensed under a Creative Commons

Attribution-NonCommercial-NoDerivs 3.0 Unported License. To view a copy of this license, visit http://creativecommons.org/licenses/by-nc-nd/3.0/

How to cite this article: Zheng, Y., He, Z., Gao, Y. \& Liu, J. Direct Desktop

Printed-Circuits-on-Paper Flexible Electronics. Sci. Rep. 3, 1786; DOI:10.1038/srep01786 (2013) 